\title{
Primary Combined Latissimus Dorsiand Serratus Anterior Flap Repair of Right-Sided Congenital Diaphragmatic Agenesis in a Neonate
}

"Madan Samuel ${ }^{1}$ and Rajiv Parapurath ${ }^{2}$

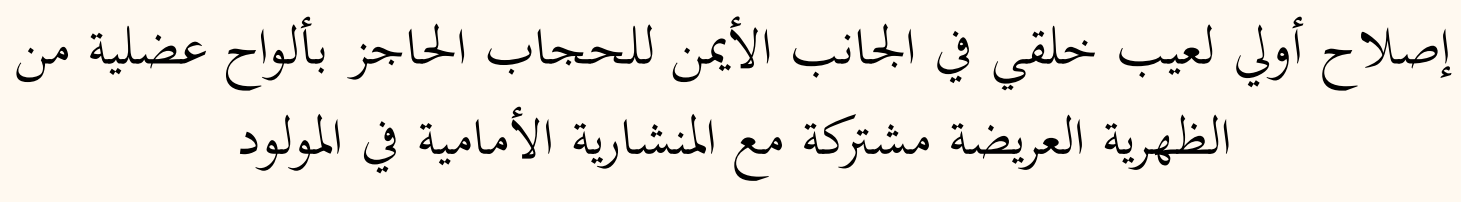

صاموئيل مادان و راجيف بارابوراث

ABSTRACT: Large diaphragmatic defects can be repaired with latissimus dorsi and serratus anterior muscle flaps. We report the first successful primary repair of complete congenital diaphragmatic agenesis using a combination of autologous living bio-tissue and synthetic mesh in a neonate born in the NMC Specialty Hospital in Dubai, United Arab Emirates, in May 2014. Poor Apgar scores, a scaphoid abdomen and absent breath sounds over the right hemithorax were observed at birth. Chest and abdominal X-rays revealed a diaphragmatic hernia. The neonate was stabilised using high-frequency oscillatory ventilation, nitric oxide and sildenafil. The right diaphragm was reconstructed using combined latissimus dorsi and serratus anterior muscle flaps reinforced by a flexible composite mesh. At 12 months old, the infant had normal respiratory function and the diaphragm was intact. No disabilities of the shoulder or scapula were observed. This case indicates that a combination of living tissue and synthetic mesh can be used to reconstruct a functional diaphragm with efficient pleuroperitoneal separation.

Keywords: Congenital Diaphragmatic Hernia; Neonate; Reconstructive Surgical Procedures; Autologous Transplantation; Surgical Mesh; Sildenafil Citrate; Case Report; United Arab Emirates.

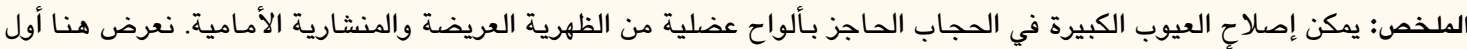

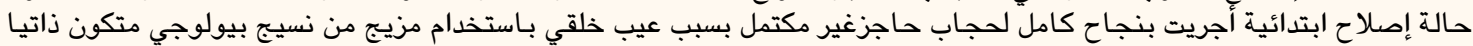

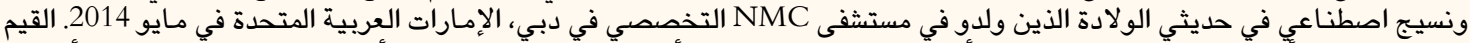

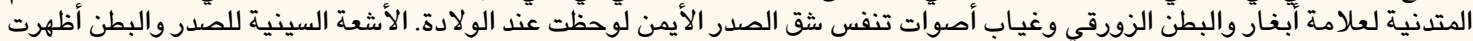

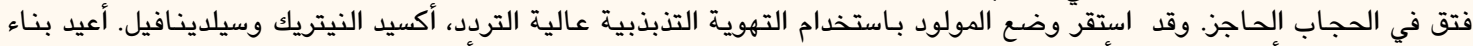

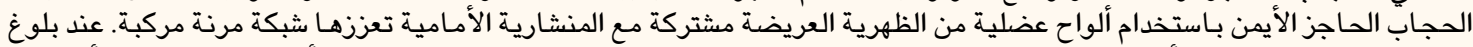

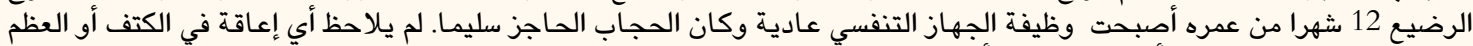

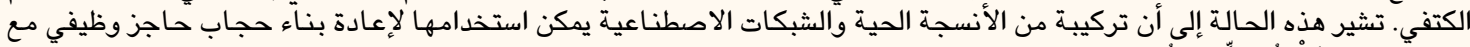

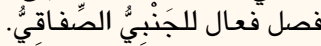

مفتاح الكلمات: فتق الحجاب الحاجز الخلقي؛ مولود؛ العمليات الجراحية الترميمية؛ زرع ذاتي؛ شبكة جراحية ؛ سيترات السيلدينافيل؛ تقرير حالة؛ الإمارات العربية المتحدة.

C ONGENITAL DiAPHRAgMATIC AGENESIS (CDA) is an extreme congenital defect which occurs due to the complete absence of the septum transversum and pleuroperitoneal membranes with an associated impaired development of the thoracic intercostal muscles. ${ }^{1,2}$ The incidence of CDA is one in 250,000 births. ${ }^{1-6}$ CDA forms a rare part of the of the congenital diaphragmatic hernia $(\mathrm{CDH})$ spectrum. In comparison, the prevalence of $\mathrm{CDH}$ is one in $2,200-2,450$ births. $^{3-6}$ The mortality rate of $\mathrm{CDH}$ is high (85-95\%) due to lung hypoplasia, pulmonary hypertension and associated cardiac anomalies..$^{3-8}$ However, the survival rate of children with large congenital diaphragmatic defects has increased due to recent advances in neonatal care. ${ }^{9}$

Reconstruction of a functional diaphragm among young patients with large diaphragmatic defects can be a difficult surgical procedure. The neodiaphragm should be an intact compliant functional muscular entity that grows with the child and ensures pleuroperitoneal separation. The disadvantages of a synthetic mesh repair include recurrence of the defect, patch migration or infection, progressive chest wall deformities and restrictive pulmonary function. ${ }^{2,6-11}$ In previous reports, diaphragms with large defects have been reconstructed in two or more stages. . $3,3,6,7,9$ 


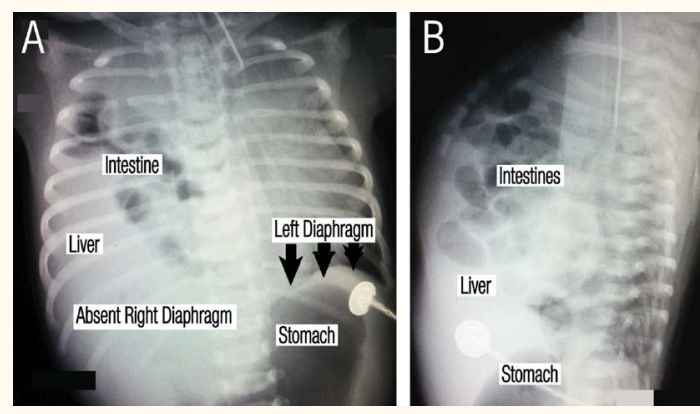

Figure 1 A\&B: Combined chest and abdomen X-rays of a male infant with right-sided congenital diaphragmatic agenesis in the (A) anteroposterior and (B) lateral aspects. A diaphragmatic hernia with herniation of the liver and intestinal loops into the right hemithorax was observed with complete absence of aeration in the right lung. The left diaphragm was intact with the stomach located in the peritoneal cavity. Note the marked mediastinal shift and impaired left lung with early signs of bronchopulmonary dysplasia.

A primary neonatal synthetic patch is usually subsequently replaced by a reversed latissimus dorsi muscle flap in childhood; the secondary reconstructed autologous living tissue flap provides a high-quality and functional diaphragm. ${ }^{2,9,12}$ Primary reconstruction of a diaphragm utilising combined latissimus dorsi and serratus anterior muscle flaps on a matrix of flexible composite mesh has not yet been reported in the literature. This report describes the preoperative stabilisation and primary reconstruction of the diaphragm of a neonate with complete CDA utilising living autologous bio-tissue and synthetic mesh.

\section{Case Report}

A 26-year-old primigravida woman gave birth at 39 gestational weeks to a full-term male infant weighing 2,580 g via supervised vaginal delivery at the NMC Specialty Hospital in Dubai, United Arab Emirates, in May 2014. The infant was born to consanguineous parents who were first cousins with an inbreeding coefficient of $>0.0156$. Poor Apgar scores, a scaphoid abdomen and absent breathing sounds over the right hemithorax were observed at birth. Combined chest and abdominal X-rays revealed a right-sided diaphragmatic hernia [Figure 1]. Cytogenetic analysis indicated a normal male karyotype $(46, \mathrm{XY})$. Further neonatal and metabolic screening tests were also normal.

Despite optimal mechanical ventilation, preductal partial pressure of oxygen and carbon dioxide in the arterial blood were 6.6-7.8 kilopascals $(\mathrm{kPa})$ and $\geq 6.0 \mathrm{kPa}$, respectively. The persistent hypoxia was associated with severe pulmonary hypertension with a three-dimensional (3D) echocardiogram showing

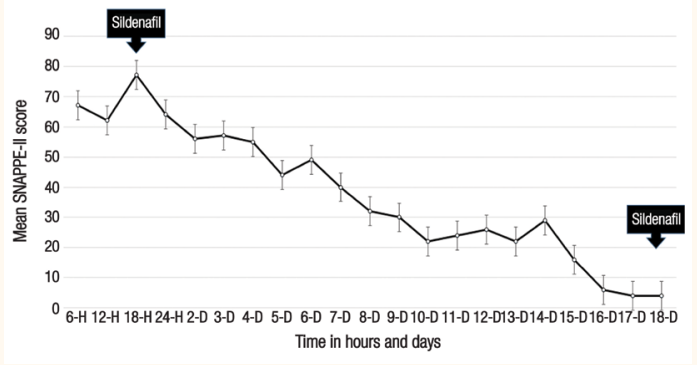

Figure 2: Illness severity and mortality risk" of a male infant with right-sided congenital diaphragmatic agenesis between six hours and 18 days since birth.

SNAPPE-II = Score for Neonatal Acute Physiology-Perinatal Extension-II; $H$ = hours; $D=$ days.

"Illness severity and mortality risk were assessed using the SNAPPE-II instrument with high scores correspondingly reflecting high illness severity and risk of mortality. ${ }^{13}$

a mean pulmonary artery versus mean systemic artery pressure ratio of $>1$. The illness severity and mortality risk of the neonate was assessed using the validated Score for Neonatal Acute PhysiologyPerinatal Extension-II (SNAPPE-II) [Figure 2]. ${ }^{13}$ At six hours old, the infant required high-frequency oscillatory ventilation and 20 parts per million of inhaled nitric oxide due to persistent hypoxia, severe pulmonary hypertension and a high SNAPPE-II score (67). Haemodynamic instability necessitated inotropic support with dobutamine $(10.0 \mu \mathrm{g} / \mathrm{kg} /$ minute $)$ and dopamine $(20.0 \mu \mathrm{g} / \mathrm{kg} /$ minute). A high oxygenation index of $>45$ prompted the administration of sildenafil (1 $\mathrm{mg} / \mathrm{kg}$ every six hours). Subsequently, a postloading dose infusion of $0.6 \mu \mathrm{g} / \mathrm{kg} /$ minute of milrinone was administered. The neonate was gradually stabilised over a period of 15 days and was weaned onto conventional mechanical ventilation by the age of 18 days.

The neonate underwent a right-sided thoracotomy through the eighth intercostal space at 22 days old. The three lobes of the right lung were atelectatic, hypoplastic and consolidated. The diaphragm was absent in the anterior, posterior and lateral aspects. The phrenic nerve was identified along the pericardium and was traced to the small fibrous rim of tissue in the medial aspect of the posterior costophrenic recess and subsequently preserved [Figure $3 \mathrm{~A}$ ]. The right adrenal gland, kidney, liver and small and large bowel formed the hernial contents. The appendix had herniated through the mesoappendix causing a partial cecal volvulus and was attached to the posterior chest wall by fibrovascular adhesions. Adhesiolysis freed the inflamed appendix and caecum and an appendectomy was performed. The non-rotated intestines and solid viscera were returned to the peritoneal cavity. 

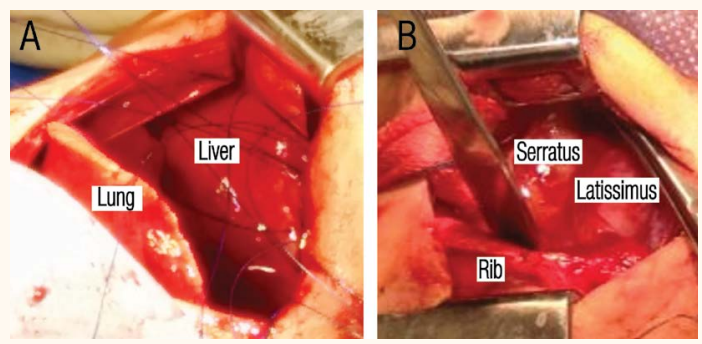

Figure 3 A\&B: Perioperative photographs of a male infant with right-sided congenital diaphragmatic agenesis demonstrating the (A) absent right diaphragm with a small fibrotic oesophageal mesenteric rim in the medial aspect and (B) reconstruction of the diaphragm with serratus anterior and latissmus dorsi flaps.

The dermo-adipose skin flaps were raised to allow visualisation of the right hemithorax and the neurovascular and muscular anatomy. The head of the latissimus dorsi was transected and the muscle was freed from the chest wall. The thoracodorsal artery, vein and nerve were identified and maintained with the flap. The latissimus dorsi flap also had an intact wide-based vascular pedicle observed through the lumbar perforators. Following the harvesting of the latissimus dorsi, the thoracodorsal vascular pedicle supplying the lower three slips of the serratus anterior muscle was observed along with the long thoracic nerve. The slips were exposed from their origin on the inferior angle of the scapula to the rib insertions. The artery and nerve to the serratus were dissected proximally and mobilised by intrafascicular dissection. The thoracodorsal artery was divided into two large branches. These two branches, the thoracodorsal vein and the circumflex scapular vascular bundle were identified and preserved. The lower edge of the serratus was delineated at the ninth rib. The posterior plane was developed up to the angle of the scapula. Posterior insertions of the muscle to the scapula and anterior insertions to the seventh, eighth and ninth ribs were detached using monopolar cautery. The absence of a distinct plane required cauterisation of several small perforating vessels from the intercostal muscles. This allowed the isolation of the major vascular pedicle $(\geq 15 \mathrm{~cm})$ up to the subcapular artery. The superior border of the $10^{\text {th }}$ rib was retracted and the intercostal muscles were freed.

A combined tension-free flap was placed into the chest through the space created. To avoid the possibility of pedicle torsion, the latissimus dorsi and serratus anterior tissue formed the posterolateral dorsal and anterolateral ventral aspects of the neo-diaphragm, respectively [Figure 3B]. A flexible composite mesh (Ethicon PHYSIOMESH ${ }^{\circledR}$ \#PHY1515Q, Johnson \& Johnson Medical GmbH, Norderstedt, Germany) formed the matrix. The composite muscle and mesh

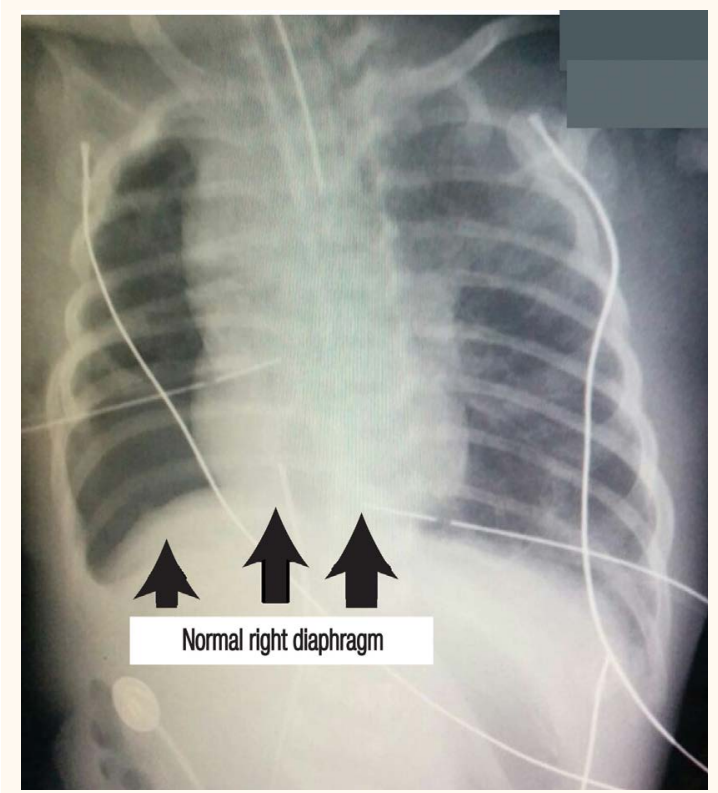

Figure 4: Fluoroscopy image showing the small hypoplastic right lung and normal domed right diaphragm of a male infant following reconstruction of the diaphragm due to right-sided congenital diaphragmatic agenesis. Note that the mediastinum has returned to the midline, which is a normal feature of post-diaphragmatic repairs.

were sutured to the periosteum using 3-0 nonabsorbable polypropylene sutures with a double-loop mattress interrupted suture technique. ${ }^{14}$ The suture line was reinforced by subcostal anchoring sutures. An end-to-side neural co-optation of the thoracodorsal nerve and phrenic nerve was performed using 9-0 polypropylene sutures. The nerve supply to the upper slips of the serratus anterior was meticulously maintained throughout the reconstruction in order to prevent winging of the scapula.

The postoperative recovery period was uneventful and the neonate was extubated on the third postoperative day. At two months of age, he was breathing room air with normal respiratory function and had achieved full oral feeds. Administration of sildenafil was tapered and subsequently stopped when the infant was three months old. By the time he was 12 months old, neurological developmental, hearing and ophthalmic evaluations were normal. Anthropometric measurements of weight $\left(50^{\text {th }}\right.$ percentile), length $\left(75^{\text {th }}\right.$ percentile) and head circumference $\left(75^{\text {th }}\right.$ percentile) were all age-appropriate. Results of the Denver Developmental Screening Test II were normal ( $75^{\text {th }}$ percentile). An intact right hemidiaphragm with uniform non-paradoxical movements during respiration and complete right lung expansion was observed via ultrasonography and fluoroscopy [Figure 4]. Electromyography showed normal function of the skeletal muscles with a phrenic nerve conduction 
velocity of 20-22 metres/second. The heart was normal as per a 3D echocardiograph. There was no winging of the scapula or any other chest deformities and the right shoulder and arm had normal function.

\section{Discussion}

CDA is a rare anomaly occurring in $5 \%$ of neonates diagnosed with $\mathrm{CDH}^{2-7,9}$ In $98 \%$ of reported cases, CDA is a left-sided defect with a high mortality rate of $85-95 \% .^{3-7}$ Right-sided CDA has a high mortality rate in comparison to right-sided $\mathrm{CDH}$ (95-98\% versus $50-70 \%) .^{2-7,9,10}$ The present report details a unique case of right-sided CDA with complete hemidiaphragmatic agenesis associated with impaired development of the thoracic intercostal muscles and oesophageal mesentery. The defect was repaired using an autologous living bio-tissue flap to facilitate the development of a compliant neo-diaphragm that would provide effective pleuroperitoneal separation.

In the current case, preoperative stabilisation of the patient was enhanced by the use of selected vasodilators and phosphodiesterase type five and type three enzyme inhibitors. These agents increase the levels of cyclic guanosine monophosphate, resulting in vascular smooth muscle relaxation and increased pulmonary blood flow. ${ }^{15}$ The synergistic action of inhaled nitric oxide, sildenafil and milrinone therefore improve circulatory haemodynamics. ${ }^{15}$ The uncomplicated postoperative recovery course was likely due to improved lung angiogenesis and alveolar growth in association with a compliant diaphragm. Sildenafil may also have prevented any worsening of the contralateral bronchopulmonary dysplasia. ${ }^{15}$

In most instances, large diaphragmatic defects in neonates are primarily repaired using a synthetic mesh which invariably leads to recurrence due to mesh migration, abscess formation/sepsis due to infection, progressive chest wall deformities due to mesh constriction or deterioration of pulmonary function due to noncompliance. ${ }^{2,9-12}$ Synthetic durable patches and acellular collagen matrices that can be remodelled by the host tissue cannot be used as neo-diaphragms due to poor compliance. ${ }^{16}$

Primary reconstruction with living bio-tissue avoids the complications of synthetic mesh repairs. The serratus anterior muscle originates from the lateral scapula and fans anteriorly into the first nine ribs. A medium-sized flap can be obtained by harvesting the lower three slips which have an independent blood and nerve supply. The latissimus dorsi and serratus anterior muscle flaps are nourished by a substantial anatomically reliable subscapular-thoracodorsal vas- cular pedicle and lumbar perforators. Double-loop mattress interrupted sutures also help to form a compliant resilient hemidiaphragm. ${ }^{14}$ Approximately $25 \%$ of the population have two thoracodorsal arteries. ${ }^{17}$ In the current case, this phenomenon may have helped to sustain good vascularity to the harvested flaps. Neuroanastomosis between the phrenic and the thoracodorsal nerve also ensured function. Additionally, the domed neo-diaphragm was both vascular and tensile to ensure growth and retain compliance and function, respectively; this resulted in efficient pleuroperitoneal separation and adequate cardiopulmonary function. Utilising a similar procedure with left-sided latissimus dorsi and serratus anterior muscle flaps may potentially repair left-sided CDA.

\section{Conclusion}

This is the first report of a successful primary reconstruction utilising combined latissimus dorsi and serratus anterior muscle flaps on a matrix of flexible composite mesh in a neonate with complete right-sided CDA. At a 12-month follow-up, the neodiaphragm was functional and the infant had no disabilities of the shoulder or scapula.

\section{References}

1. Tsang TM, Tam PK, Dudley NE, Stevens J. Diaphragmatic agenesis as a distinct clinical entity. J Pediatr Surg 1995;30:16-18. doi: 10.1016/0022-3468(95)90599-5.

2. Samarakkody U, Klaassen M, Nye B. Reconstruction of congenital agenesis of hemidiaphragm by combined reversed latissimus dorsi and serratus anterior muscle flaps. J Pediatr Surg 2001; 36:1637-40. doi: 10.1053/jpsu.2001.27937.

3. Neville HL, Jaksic T, Wilson JM, Lally PA, Hardin WD Jr, Hirschl RB, et al. Bilateral congenital diaphragmatic hernia. J Pediatr Surg 2003; 38:522-4. doi: 10.1053/jpsu.2003.50092.

4. Ruano R, Takashi E, da Silva MM, Campos JA, Tannuri U, Zugaib M. Prediction and probability of neonatal outcome in isolated congenital diaphragmatic hernia using multiple ultrasound parameters. Ultrasound Obstet Gynecol 2012; 39:42-9. doi: 10.1002/uog.10095.

5. Tudorache S, Chiuțu LC, Illiescu DG, Georgescu R, Stoica GA, Simionescu CE, et al. Prenatal diagnosis and perinatal outcome in congenital diaphragmatic hernia: Single tertiary center report. Rom J Morphol Embryol 2014; 55:823-33.

6. Jani P, Bidarkar SS, Walker K, Halliday R, Badawi N, Cohen RC. Right-sided congenital diaphragmatic hernia: A tertiary centre's experience over 25years. J Neonatal Perinatal Med 2014; 7:39-45. doi: 10.3233/NPM-1474313.

7. Fisher JC, Jefferson RA, Arkovitz MS, Stolar CJ. Redefining outcomes in right congenital diaphragmatic hernia. J Pediatr Surg 2008; 43:373-9. doi: 10.1016/j.jpedsurg.2007.10.049.

8. Koziarkiewicz M, Taczalska A, Piaseczna-Piotrowska A. Longterm follow-up of children with congenital diaphragmatic hernia: Observations from a single institution. Eur J Pediatr Surg 2014; 24:500-7. doi: 10.1055/s-0033-1357751. 
9. Barbosa RF, Rodrigues J, Correia-Pinto J, Costa-Ferreira A, Cardoso A, Reis JC, et al. Repair of a large congenital diaphragmatic defect with a reverse latissimus dorsi muscle flap. Microsurgery 2008; 28:85-8. doi: 10.1002/micr.20455.

10. Zardo P, Zhang R, Wiegman B, Haverich A, Fischer S. Biological materials for diaphragmatic repair: Initial experience with the PeriGuard Repair Patch ${ }^{\circledR}$. Thorac Cardiovasc Surg 2011; 59:40-4. doi: 10.1055/s-0030-1250499.

11. Bekdash B, Singh B, Lakhoo K. Recurrent late complications following congenital diaphragmatic hernia repair with prosthetic patches: A case series. J Med Case Rep 2009; 26:7237. doi: 10.1186/1752-1947-3-7237.

12. Sydorak RM, Hoffman W, Lee H, Yingling CD, Longaker M, Chang J, et al. Reversed latissimus dorsi muscle flap for repair of recurrent congenital diaphragmatic hernia. J Pediatr Surg 2003; 38:296-300. doi: 10.1053/jpsu.2003.50097.

13. Richardson DK, Corcoran JD, Escobar GJ, Lee SK. SNAPII and SNAPPE-II: Simplified newborn illness severity and mortality risk scores. J Pediatr 2001; 138:92-100. doi: 10.1067/ mpd.2001.109608.
14. Biddlestone J, Samuel M, Creagh T, Ahmad T. The double loop mattress suture. Wound Repair Regen 2014; 22:415-23. doi: 10.1111/wrr.12159.

15. Noori S, Friedlich P, Wong P, Garingo A, Seri I. Cardiovascular effects of sildenafil in neonates and infants with congenital diaphragmatic hernia and pulmonary hypertension. Neonatology 2007; 91:92-100. doi: 10.1159/000097125.

16. Mayer S, Decaluwe H, Ruol M, Manodoro S, Kramer M, Till H, et al. Diaphragm repair with a novel cross-linked collagen biomaterial in a growing rabbit model. PLoS One 2015 10:e0132021. doi: 10.1371/journal.pone.0132021.

17. Majumdar S, Bhattacharya S, Chatterjee A, Dasgupta $\mathrm{H}$ Bhattacharya K. A study on axillary artery and its branching pattern among the population of West Bengal, India. Ital J Anat Embryol 2013; 118:159-71. 